\title{
Double Helix Magnetic Order in CrAs with MnP-Type Crystal Structure
}

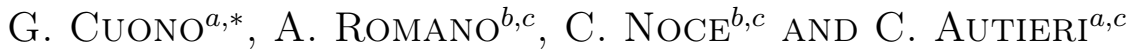 \\ ${ }^{a}$ International Research Centre Magtop, Institute of Physics, Polish Academy of Sciences, \\ Aleja Lotnikow 32/46, PL-02668 Warsaw, Poland \\ ${ }^{b}$ Dipartimento di Fisica "E.R. Caianiello", Università degli Studi di Salerno, \\ I-84084 Fisciano (SA), Italy \\ ${ }^{c}$ Consiglio Nazionale delle Ricerche CNR-SPIN, UOS Salerno, I-84084 Fisciano (Salerno), Italy
}

Received: 29.10.2021 \& Accepted: 05.12.2021

Doi: 10.12693/APhysPolA.141.35

*e-mail: gcuono@magtop. ifpan.edu.pl

\begin{abstract}
The transition metal pnictide CrAs exhibits superconductivity in the vicinity of a helimagnetic phase, where it has been found that the propagation vector is parallel to the $c$-axis and the magnetic moments lie in the $a-b$ plane. Here we use $a b$ initio calculations to study the magnetic interactions in the material. Mapping onto a Heisenberg Hamiltonian, we calculate the magnetic exchanges with LDA $+U$ calculations and we unveil the origin of the magnetic frustration. Finally, we reproduce the double helix magnetic order with the propagation vector $\boldsymbol{Q}=(0,0,0.456)$ and we obtain the magnetic transition temperature $T_{\mathrm{N}}$ through Monte Carlo simulations of the specific heat. Due to the limitations of the use of the Heisenberg Hamiltonian for itinerant magnetic systems, the theoretical $T_{\mathrm{N}}$ underestimated the experimental value of the pure CrAs. However, our results are in good agreement with those found for the alloy $\mathrm{CrAs}_{0.5} \mathrm{Sb}_{0.5}$ belonging to the same space group, showing that our result can describe this material class.
\end{abstract}

topics: Cr-based superconductors, magnetic frustration, double helix

\section{Introduction}

In recent years an increasing interest has been devoted to transition metal pnictides $[1,2]$. These materials show unconventional properties determined by the existence of a superconducting phase which develops in proximity of a magnetic one that can be suppressed through the application of a physical or a chemical pressure [3-6]. Some materials of this family, such as CrAs [3, 5-11], MnP [12] and WP [13-15], belong to the space group Pnma. In turn, another family is represented by the compounds $\mathrm{A}_{2} \mathrm{Cr}_{3} \mathrm{As}_{3}$ [16-21], with the element $\mathrm{A}$ being $\mathrm{Na}$ [22], K [16], Rb [23] or Cs [24], which in common with the first group show the possible presence of unconventional superconductivity. In particular, in the chemical cation deintercalation process applied to $\mathrm{K}_{2} \mathrm{Cr}_{3} \mathrm{As}_{3}$ to obtain the magnetic parent compound $\mathrm{KCr}_{3} \mathrm{As}_{3}$, an intermediate state between 233 and 133 phases has been observed [25, 26], possibly allowing to tune the interplay between the magnetic and superconducting properties [27]. Generally speaking, we can say that though these systems are being thoroughly investigated to date, they still need a careful analysis to better understand the interplay between structural, electronic, magnetic, and superconducting properties and their connections to topological features.
The material class with Pnma phase (space group number 62) presents nonsymmorphic symmetries that are responsible for additional degeneracies of the bands $[14,18]$. This affects the shape of the Fermi surface as well as the magnetic and the superconducting properties, giving rise, together with the presence of strong magnetic fluctuations, to a quasilinear magnetoresistance (MR) [28] near magnetic instability. There are very few cases of non-compensated materials with topological band structure showing large MR [29], while a non-saturating extremely large MR due to the presence of compensated Fermi pockets is more common in nature [30]. Regarding CrAs, pressureinduced superconductivity was found at $T_{c} \approx 2 \mathrm{~K}$ and $P_{c} \approx 8 \mathrm{kbar}$ in proximity of a helimagnetic (HM) phase $[3,6]$. In the non-collinear HM one, it has been found by neutron diffraction measurements $[31,32]$ that the propagation vector is parallel to the $c$-axis and the magnetic moments lie in the $a-b$ plane. The transition to the HM phase occurs at $T_{\mathrm{N}} \approx 275 \mathrm{~K}$. Four spirals propagating along the $c$-axis with a well-defined angle between them form the magnetic structure of the compound, and the magnetic moment decreases with pressure from $1.7 \mu_{\mathrm{B}}$ to $0.4 \mu_{\mathrm{B}}$ at a pressure of $0.7 \mathrm{GPa}$, when superconductivity starts to appear and the magnetic order is suppressed [32]. This decrease of the 
magnetic moment is accompanied by a spin reorientation, from a configuration in which the magnetic moments lie in the $a-b$ plane to one in which they lie in the $a-c$ plane [31]. We recall that in these compounds the magnetism is due to the $d$ orbitals of the $\mathrm{Cr}$ atoms at the Fermi level [7-9].

In CrAs, the magnetic, structural, and electronic properties are intimately related, as made evident by the fact that the magnetic transition is accompanied by discontinuous changes of the lattice parameters [3]. These effects have been analyzed via $a b$ initio studies [7] and model Hamiltonian investigations [8-10], which allowed to conclude that $\mathrm{CrAs}$ is a metal with moderate electronic correlations. Indeed, the band structure is well described within the density functional theory (DFT) [7], and a relatively small value of the Coulomb repulsion allows for the magnetic moment to obtain a value consistent with the experimental one [8].

The non-collinear magnetic states can originate from the Dzyaloshinskii-Moriya interaction or from magnetic frustrations. Several examples of the latter are present in transition metal compounds [33-36]. Depending on the symmetries and degree of frustration, the system can show spin-spiral, double helix, spin glass or a collinear magnetic phase in case of low frustration. In particular, many transition metal monopicnitides show double helix magnetic order [4], with this kind of order having been recently found also in CrAs. From the inelastic neutron scattering data on polycrystalline samples, an oversimplified model for exchange couplings was derived in order to demonstrate the origin of the magnetic frustration of the double spin helix [37]. However, a study within DFT of the magnetic frustration and of the double helix order is still missing in the literature.

In this paper, we improve the theoretical description of the double helix configuration in CrAs presented in [37] by calculating the magnetic couplings via $a b$ initio $\mathrm{DFT}+U$ method mapped onto a Heisenberg model. We unveil the origin of the magnetic frustration that originates the double helix, reliably simulating the related helical magnetic order. The validity of our approach has been tested by evaluating the specific heat and the transition temperature $T_{\mathrm{N}}$ through the Monte Carlo method. We show that although the system is metallic, the simple Heisenberg model allows to capture fundamental properties such as the frustration between magnetic exchanges of different nature and the double helical structure.

The paper is organized as follows: in Sect. 2 we report the computational details, in Sect. 3 - the results and in Sect. 4 - conclusions.

\section{Computational details}

We have performed density functional theory calculations by using the VASP package [38-41]. In this approach, the core and the valence electrons have been treated within the projector augmented wave (PAW) method [42] and with a cutoff of $400 \mathrm{eV}$ for the plane wave basis. All the calculations have been performed using a $12 \times 16 \times 10$ $k$-point grid. For the treatment of the exchange correlation, the local density approximation (LDA) and the Perdew-Zunger parametrization [43] of the Ceperley-Alder [44] data have been considered, as these usually give better results for itinerant magnetic systems with magnetic moment far from highspin configuration [45-47]. In DFT, the electronic interactions are already present as the sum of the Hartree term and the exchange correlation that include all the correlations and spin interactions. When weakly correlated compounds are studied, it is not necessary to consider additional interactions. Instead, when moderately and strongly correlated electron systems are considered, an additional Coulomb repulsion $U$ is added to the energy functional [48]. This leads to an increase of the magnetic moment with respect to what one finds with the simple LDA. Therefore, in the case of moderate correlations, the $U$ values must be carefully chosen in order to avoid an overestimation of the magnetic moment. For the system analyzed here, we have found that the best agreement with the experiments is obtained for $U=1 \mathrm{eV}$.

In the evaluation of the magnetic exchanges of CrAs, we found that not all the magnetic configurations converge in the case of collinear magnetism. However, the inclusion of the spin-orbit coupling favored the convergence, allowing the determination of a set of magnetic configurations sufficient to obtain the relevant exchange couplings needed to describe the double helix magnetic order.

\section{Results}

In this section, we first present the calculation of the magnetic couplings and then we analyze the double-helix magnetic order in CrAs, evaluating in particular the specific heat by use of Monte Carlo simulations.

\subsection{Electronic properties and magnetic exchanges}

CrAs belongs to the family of transition-metal pnictides sharing the general formula $\mathrm{AB}$ (A means transition metal, $\mathrm{B}=\mathrm{P}, \mathrm{As}, \mathrm{Sb})$. It exhibits either a hexagonal NiAs-type (B81) structure or an orthorhombic MnP-type (B31) structure. In the latter case, the unit-cell lattice parameters are $a=5.649 \AA, b=3.463 \AA$ and $c=6.2084 \AA$. The chromium atoms are situated in the center of the $\mathrm{CrAs}_{6}$ octahedra, surrounded by six nearestneighbour arsenic atoms, as shown in Fig. 1. Four of the six $\mathrm{Cr}-\mathrm{As}$ bonds are inequivalent due to the high anisotropy exhibited by this class of compounds.

The band structure of CrAs obtained in LDA [7] is characterized by a bandwidth of $3.5 \mathrm{eV}$. We can compare this value with the one of the $t_{2 g}$ bands in strongly correlated Cr-based systems, 


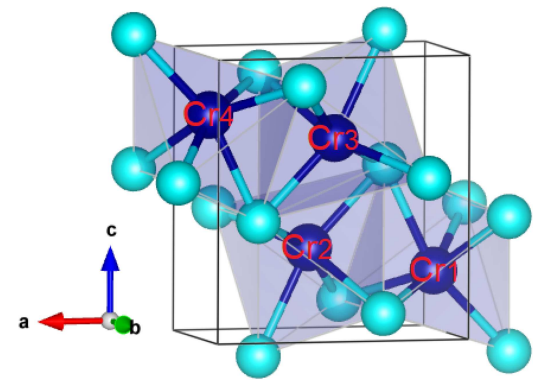

Fig. 1. Crystal structure of CrAs in the MnP-type phase, and $\mathrm{Cr}$ and As atoms are shown as blue and cyan spheres, respectively. The $\mathrm{CrAs}_{6}$ face-shared octahedra are transparent. The solid black lines represent the orthorhombic primitive unit cell.

equal to $2.5 \mathrm{eV}$ [47-49], as well as with the one of the uncorrelated elemental $\mathrm{Cr}$ which is around $5.0 \mathrm{eV}[50,51]$. This allows to conclude that CrAs is a moderately correlated system. We have thus performed $a b$ initio LDA $+U$ calculations scanning a range of low values of $U$, eventually obtaining the best agreement with the experimental data on the exchange couplings for $U=1 \mathrm{eV}$.

We have used the Hamiltonian [37]

$$
H=\sum_{i, j} J_{i j} \boldsymbol{S}_{i} \cdot \boldsymbol{S}_{j}
$$

considering the first-neighbours exchange couplings, denoted as $J_{a}, J_{b}, J_{c 1}, J_{c 2}$, the in-plane secondneighbour coupling $J_{S N p}$, and the second-neighbour couplings along $c$-axis, i.e., $J_{S N c 1}$ and $J_{S N c 2}$. All these are shown in Fig. 1a.

The collinear magnetic phase closer to the doublehelix ground state was investigated before and found to be of G-type [8, 9]. Here we have considered the ferromagnetic phase together with three other magnetic phases with zero net magnetic moment which are usually considered in G-type, C-type and A-type Pnma perovskites. With reference to the eight-atom unit cell, the four equations for the mentioned above four magnetic configurations are

$$
\begin{aligned}
& E_{F M}=E_{1}+4 J_{a}+4 J_{c 1}+4 J_{c 2}+8 J_{S N p} \\
& \quad+8 J_{S N c 1}+8 J_{S N c 2}, \\
& E_{G}=E_{1}-4 J_{a}-4 J_{c 1}-4 J_{c 2}-8 J_{S N p} \\
& \quad+8 J_{S N c 1}+8 J_{S N c 2}, \\
& E_{C}=E_{1}-4 J_{a}+4 J_{c 1}+4 J_{c 2}-8 J_{S N p} \\
& \quad-8 J_{S N c 1}-8 J_{S N c 2}, \\
& E_{A}=E_{1}+4 J_{a}-4 J_{c 1}-4 J_{c 2}+8 J_{S N p} \\
& \quad-8 J_{S N c 1}-8 J_{S N c 2},
\end{aligned}
$$

where $E_{1}$ is a trivial constant energy and the other quantities are the magnetic exchanges, shown in Fig. 2a. In (2)-(5) we have seven unknowns

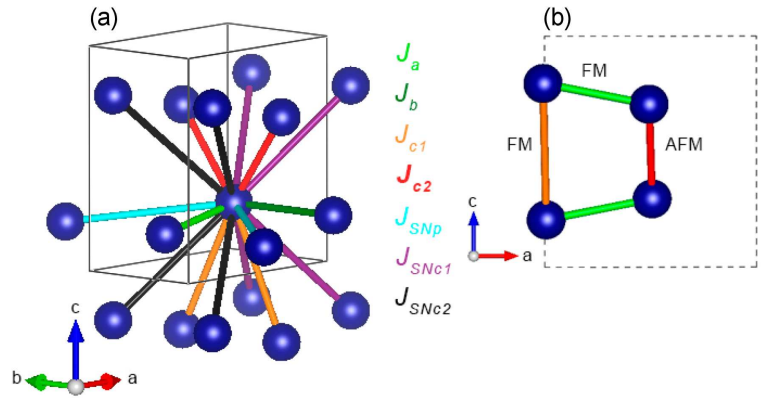

Fig. 2. Magnetic couplings considered in our calculation. In part (a) we show the $3 \mathrm{D}$ sight (the colours of the $J$ denominations coincide with the bonds colours). In part (b) we show the characteristic $\mathrm{Cr}-\mathrm{Cr}$ distances in the $a-c$ plane. The ferromagnetic coupling is indicated with FM, while the antiferromagnetic one - with AFM.

quantities, not including $J_{b}$. Moreover, we can only estimate the quantity $J_{c 1}+J_{c 2}$, but not the single $J_{c 1}$ and $J_{c 2}$ couplings.

The DFT calculations do not converge easily once we go away from the G-type magnetic configuration or away from the zero magnetic moment ones. Therefore, to estimate $J_{b}$, we have doubled the unit cell along the $b$-axis, but taken only the configurations with zero net magnetic moment. In particular, we have considered two unit cells with G-type magnetic order, one coupled ferromagnetically and the other one antiferromagnetically. The related energies being denoted as $E_{\mathrm{GFM} b}$ and $E_{\mathrm{GAFM}}$. We have repeated the same calculation for the A-type configuration coupled antiferromagnetically along the $b$-axis (the energy is $E_{\mathrm{AAFM} b}$ ), in this way obtaining

$$
\begin{gathered}
E_{\mathrm{GFM} b}=2 E_{2}-8 J_{a}+8 J_{b}-8 J_{c 1}-8 J_{c 2} \\
-16 J_{S N p}+16 J_{S N c 1}+16 J_{S N c 2}, \\
E_{\mathrm{GAFM} b}=2 E_{2}-8 J_{a}-8 J_{b}+16 J_{S N p}, \\
E_{\mathrm{AAFM} b}=2 E_{2}+8 J_{a}-8 J_{b}-16 J_{S N p} .
\end{gathered}
$$

The quantity $E_{2}$ is also a trivial constant energy, but its value is different from $E_{1}$.

To estimate $J_{c 1}+J_{c 2}$, we have doubled the unit cell along the $c$-axis considering two unit cells with G-type magnetic order, again coupled ferromagnetically and antiferromagnetically, respectively. We define their energies as $E_{\mathrm{GFMc}}$ and $E_{\mathrm{GAFM}}$. Thus, we have the following equations

$$
\begin{aligned}
& E_{\mathrm{GAFM} c}=2 E_{3}+8 J_{c 1}-8 J_{c 2}, \\
& E_{\mathrm{GFM} c}=2 E_{3}-8 J_{c 1}+8 J_{c 2} .
\end{aligned}
$$

The quantity $E_{3}$ is again a trivial constant energy, different from $E_{1}$ and $E_{2}$.

Solving the previous equations (2)-(10), we have mapped the $a b$ initio results onto a Heisenberg model [55] reporting the magnetic exchanges values 
TABLE I

In the first row of the table, we indicate the distances in $[\AA]$ between neighboring atoms, in the third and the fourth row we report the values of $J$ obtained with our calculations in LDA $+U$ approximation and the values from [37], respectively. The parameters $J_{a}, J_{b}, J_{c 1}$ and $J_{c 2}$ refer to the first neighbours along $a$-, $b$ - and $c$-axes; $J_{S N p}$ to the in-plane second neighbour; $J_{S N c 1}$ and $J_{S N c 2}$ refer to the second neighbours along $c$-axis. The latter are two independent parameters which in our approximation are assumed to be equal. Since they are not the dominant exchange couplings, this approximation will not influence the main results. The values of $J$ are expressed in [meV], while the one of the Coulomb repulsion is in $[\mathrm{eV}]$.

\begin{tabular}{c|c|c|c|c|c|c|c}
\hline \hline & \multicolumn{3}{|c|}{ First neighbours } & \multicolumn{3}{c}{ Second neighbours } \\
\hline distance & 2.857 & 3.588 & 4.042 & 3.090 & 4.586 & 4.478 & 4.574 \\
\hline & $J_{a}$ & $J_{b}$ & $J_{c 1}$ & $J_{c 2}$ & $J_{S N p}$ & $J_{S N c 1}$ & $J_{S N c 2}$ \\
\hline LDA $+U$ & -19.4 & -25.6 & -21.9 & 58.6 & 5.6 & -8.2 & -8.2 \\
Ref. [37] & 9.27 & & -4.82 & 65.0 & & &
\end{tabular}

in Table I. We find that $J_{c 2}$ is the largest exchange coupling and it is ferromagnetic, while all the other nearest-neighbour ones are antiferromagnetic. Smaller values are found for the secondnearest neighbour couplings, as expected, with inplane and out-of-plane ones which are respectively ferromagnetic and antiferromagnetic. We point out that these results come from an energy minimization procedure and thus cannot be directly compared with the ones reported in [37] where the exchange couplings were treated rather as fitting parameters. We also notice that though this kind of mapping has limitations for metals, the large $T_{\mathrm{N}}$ value of CrAs should ensure that the obtained results are correct.

\subsection{Double helix magnetic order}

Using the Uppsala atomistic spin dynamics (UppASD) package [53], we have performed the Monte Carlo simulations to evaluate the specific heat, considering a $16 \times 16 \times 16$ supercell and referring to the Heisenberg-like Hamiltonian (1). In $\mathrm{CrAs}$ there are two different characteristic $\mathrm{Cr}-\mathrm{Cr}$ distances along the $c$-axis, indicated as $\mathrm{Cr}_{2}-\mathrm{Cr}_{3}$ and $\mathrm{Cr}_{1}-\mathrm{Cr}_{4}$ in Fig. 1 and equal to $3.090 \AA$ and $4.042 \AA$, respectively. In the former case, i.e., in the case of shorter distance, the magnetic coupling is strongly antiferromagnetic, while in the latter one it is weakly ferromagnetic, as shown in Fig. 2 b.

In Fig. 2b we observe that we cannot minimize the energy at all the bonds of the quadrilateral. This leads to the magnetic frustration that is responsible for the spiral configuration. In order to reproduce the experimentally detected wave vector $\boldsymbol{Q}=(0,0,0.356)$, the stability values of the exchange coupling leading to the double helix magnetic ground state were already determined by Matsuda et al. [37] as $J_{c 2} / J_{a}=7.1$ and $J_{c 1} / J_{a}=-0.52$, irrespective of the value and sign of $J_{b}$. The magnetic couplings obtained are reported in Table I together with the values obtained in [37]. The results are different because they are obtained with two different methods, and both methods have limitations. We used $a b$ initio calculations and therefore

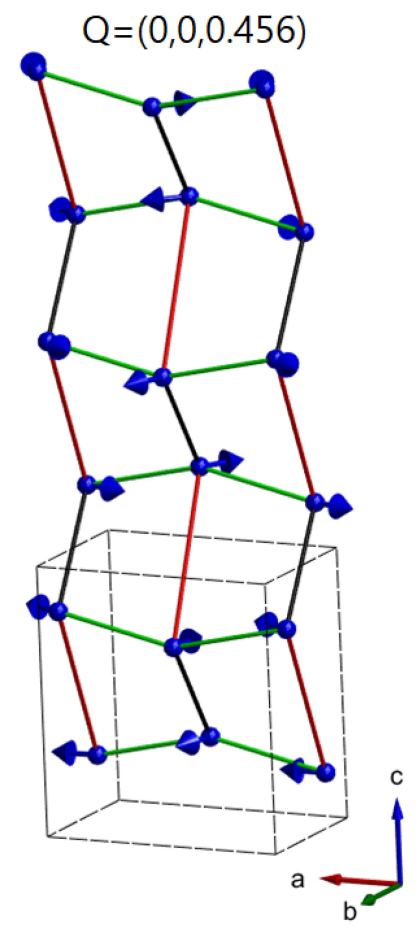

Fig. 3. Double helix structure of CrAs for $\boldsymbol{Q}=$ $(0,0,0.456)$ obtained with our data for the exchange couplings. The blue arrows represent the spin of the chromium atoms. Arsenium atoms are not shown. The grey dashed lines represent the primitive unit cell.

we do not have a perfect agreement with the experiment, while Matsuda et al. [37] made a fit to the experimental result with a $2 \mathrm{D}$ model, neglecting $J_{b}$ and the second neighbours. The experimental propagating wave vector in a reciprocal lattice unit is $\boldsymbol{Q}=(0,0,0.356)$ [37]. The wave vector obtained with the $\mathrm{LDA}+U$ values of the exchange couplings is $\boldsymbol{Q}=(0,0,0.456)$. This value of $\boldsymbol{Q}$ is actually close to that reported by Wang et al. [4] for $\mathrm{CrAs}_{x} \mathrm{Sb}_{1-x}$. The corresponding double helix structure has been calculated by means of the algorithm of [54] and is reported in Fig. 3. 


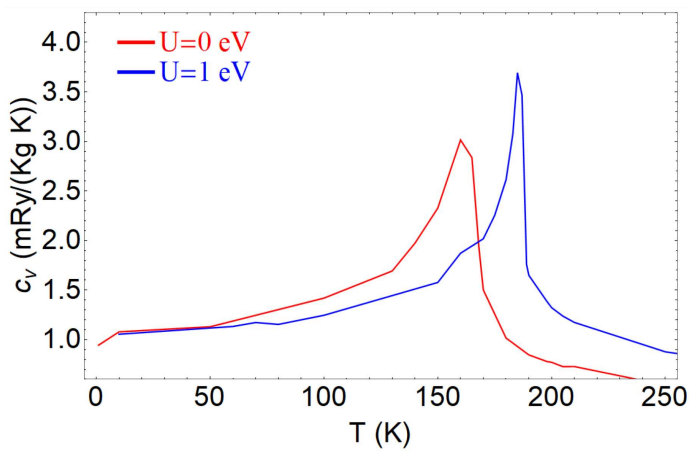

Fig. 4. The red line shows the temperature dependence of the specific heat of CrAs evaluated from the magnetic exchanges calculated in LDA, and the blue line shows the specific heat calculated in $\mathrm{LDA}+U$ approximation for $U=1 \mathrm{eV}$. For the curve at $U=0 \mathrm{eV}$, the critical temperature is $T_{\mathrm{N}}=160 \mathrm{~K}$, while for the one at $U=1 \mathrm{eV}, T_{\mathrm{N}}=185 \mathrm{~K}$.

From the $\mathrm{LDA}+U$ values obtained for the exchange couplings, we evaluated the specific heat in order to make an estimate of the transition temperature $T_{\mathrm{N}}$. The results are shown in Fig. 4 where the curves for $U=0$ and $U=1 \mathrm{eV}$ are presented. Note that for $U=1 \mathrm{eV}$ we obtain the critical temperature value $T_{\mathrm{N}}=185 \mathrm{~K}$ underestimated to the experimental one, which is equal to $T_{\mathrm{N}} \approx 265 \mathrm{~K}$. We also tried with $U=2 \mathrm{eV}$ but this choice did not lead to an improvement of the result. Therefore, we conclude that the more reliable set of couplings is the one obtained choosing $U=1 \mathrm{eV}$, reported in Table I. Although the calculated value of $T_{\mathrm{N}}$ underestimates the experimental value found in CrAs, it is nonetheless close to the value reported for $\mathrm{CrAs}_{0.5} \mathrm{Sb}_{0.5}$ [4]. We can thus say that this result, together with the one obtained for the propagation vector $\boldsymbol{Q}$, suggests that our approach allows to describe qualitatively the properties of this class of materials.

\section{Conclusions}

We have evaluated the magnetic exchanges of the pressure-induced superconductor chromium arsenide CrAs via $a b$ initio $\mathrm{LDA}+U$ calculations, and then mapping the energy of the magnetic configuration onto a Heisenberg Hamiltonian. The best agreement with the experimental results is obtained for a Coulomb repulsion $U=1 \mathrm{eV}$, providing evidence that the system is a moderately correlated compound. This finding is consistent with the fact that the band structure is well reproduced by density functional theory and that the magnetic moment value obtained for small $U$ agrees with the experimental one $[7,8]$. We have analyzed the double helix structure and, in particular, we have determined the temperature dependence of the specific heat using Monte Carlo simulations. The theoretical value of the critical temperature $T_{\mathrm{N}}$ was found to be equal to $185 \mathrm{~K}$, which underestimates the experimental value of $265 \mathrm{~K}$. This discrepancy was to some extent expected as it is well known that the use of a Heisenberg-like model alone cannot describe an itinerant magnet in a fully satisfactory way. Nonetheless, we notice that our ab initio approach allows to qualitatively reproduce the magnetic exchange couplings and the double helix structure with a propagation vector $\boldsymbol{Q}=(0,0,0.456)$. We expect that a further improvement of our results can be obtained by including in the approach presented here the dynamical effects related to the behavior of the chromium $3 d$ electrons as well as the Dzyaloshinskii-Moriya interaction. In particular, the latter may prove to be relevant due to the role played by the spin-orbit coupling in the arsenic atoms located between two chromium ones.

\section{Acknowledgments}

The work was supported by the Foundation for Polish Science through the International Research Agendas program cofinanced by the European Union within the Smart Growth Operational Programme.

\section{References}

[1] R.Y. Chen, N.L. Wang, Rep. Prog. Phys. 82, 012503 (2019).

[2] C. Noce, EPL 130, 67001 (2020).

[3] W. Wu, L. Cheng, K. Matsubayashi, P. Kong, F. Lin, C. Jin, N. Wang, Y. Uwatoko, J. Luo, Nat. Commun. 5, 5508 (2014).

[4] Y. Wang, Y. Feng, J.-G. Cheng, W. Wu, J.L. Luo, T.F. Rosenbaum, Nat. Commun. 7, 13037 (2016).

[5] W. Wu, X. Zhang, Z. Yin, P. Zheng, N. Wang, J. Luo, Sci. China Phys. Mech. Astron. 53, 1207 (2010).

[6] H. Kotegawa, S. Nakahara, H. Tou, H. Sugawara, J. Phys. Soc. Jpn. 83, 093702 (2014).

[7] C. Autieri, C. Noce, Philos. Mag. 97, 3276 (2017).

[8] C. Autieri, G. Cuono, F. Forte, C. Noce, J. Phys. Condens. Matter 29, 224004 (2017).

[9] C. Autieri, G. Cuono, F. Forte, C. Noce, J. Phys. Conf. Ser. 969, 012106 (2018).

[10] G. Cuono, C. Autieri, G. Guarnaccia, A. Avella, M. Cuoco, F. Forte, C. Noce, Eur. Phys. J. Spec. Top. 228, 631 (2019).

[11] A. Nigro, P. Marra, C. Autieri, W. Wu, J.G. Cheng, J. Luo, C. Noce, Europhys. Lett. 125, 57002 (2019).

[12] J.-G. Cheng, K. Matsubayashi, W. Wu, J.P. Sun, F.K. Lin, J.L. Luo, Y. Uwatoko, Phys. Rev. Lett. 114, 117001 (2015). 
[13] Z. Liu, W. Wu, Z. Zhao et al., Phys. Rev. B 99, 184509 (2019).

[14] G. Cuono, F. Forte, M. Cuoco, R. Islam, J. Luo, C. Noce, C. Autieri, Phys. Rev. Mater. 3, 095004 (2019).

[15] A. Nigro, G. Cuono, P. Marra et al., arXiv:2009.07849.

[16] J.-K. Bao, J.-Y. Liu, C.-W. Ma et al., Phys. Rev. X 5, 011013 (2015).

[17] G. Cuono, C. Autieri, F. Forte, G. Busiello, M.T. Mercaldo, A. Romano, C. Noce, A. Avella, AIP Adv. 8, 1 (2018).

[18] G. Cuono, C. Autieri, F. Forte, M.T. Mercaldo, A. Romano, A. Avella, C. Noce, New J. Phys. 21, 063027 (2019).

[19] G. Cuono, F. Forte, A. Romano, X. Ming, J. Luo, C. Autieri, C. Noce, Phys. Rev. B 103, 214406 (2021).

[20] G. Cuono, F. Forte, A. Romano, X. Ming, J. Luo, C. Autieri, C. Noce, Phys. Rev. Mater. 5, 064402 (2021).

[21] G. Cuono, Il Nuovo Cim. 44C, 109 (2021).

[22] Q.-G. Mu, B.-B. Ruan, B.-J. Pan, T. Liu, J. Yu, K. Zhao, G.-F. Chen, Z.-A. Ren, Phys. Rev. Mater. 2, 034803 (2018).

[23] Z.-T. Tang, J.-K. Bao, Y. Liu et al., Phys. Rev. B 91, 020506(R) (2015).

[24] Z.-T. Tang, J.-K. Bao, Z. Wang et al., Sci. China Mater. 58, 16 (2015).

[25] L. Zhang, J. Li, Q. Mu et al., J. Phys. Condens. Matter. 33, 215404 (2021).

[26] A. Galluzzi, G. Cuono, A. Romano, J. Luo, C. Autieri, C. Noce, M. Polichetti, arXiv:2107.05032.

[27] C. Autieri, M. Cuoco, C. Noce, Phys. Rev. B 85, 075126 (2012).

[28] Q. Niu, W.C. Yu, K.Y. Yip, et al., Nature Commun. 8, 15358 (2017).

[29] D.J. Campbell, J. Collini, J. Sławińska et al., npj Quantum Mater. 6, 1 (2021).

[30] A.S. Wadge, G. Grabecki, C. Autieri et al., J. Phys.: Condens. Matter 34, 125601 (2022).

[31] Y. Shen, Q. Wang, Y. Hao et al., Phys. Rev. $B$ 93, 060503(R) (2016).

[32] L. Keller, J.S. White, M. Frontzek, P. Babkevich, M.A. Susner, Z.C. Sims, A.S. Sefat, H.M. Rønnow, C. Rüegg, Phys. Rev. B 91, 020409(R) (2015).

[33] X. Ming, C. Autieri, K. Yamauchi, S. Picozzi, Phys. Rev. B 96, 205158 (2017).
[34] S.A. Ivanov, A.A. Bush, A.I. Stash et al., Inorg. Chem. 55, 2791 (2016).

[35] C. Autieri, C. Śliwa, R. Islam, G. Cuono, T. Dietl, Phys. Rev. B 103, 115209 (2021).

[36] C. Śliwa, C. Autieri, J.A. Majewski, T. Dietl, Phys. Rev. B 104, L220404 (2021).

[37] M. Matsuda, F.K. Lin, R. Yu et al., Phys. Rev. X 8, 031017 (2018).

[38] G. Kresse, J. Hafner, Phys. Rev. B 47, 558(R) (1993).

[39] G. Kresse, J. Hafner, Phys. Rev. B 49, 14251 (1994).

[40] G. Kresse, J. Furthmüller, Comput. Mater. Sci. 6, 15 (1996).

[41] G. Kresse, J. Furthmüller, Phys. Rev. B 54, 11169 (1996).

[42] G. Kresse, D. Joubert, Phys. Rev. B 59, 1758 (1999).

[43] J.P. Perdew, A. Zunger, Phys. Rev. B 23, 5048 (1981).

[44] D.M. Ceperley, B.J. Alder, Phys. Rev. Lett. 45, 566 (1980).

[45] G. Cuono, C. Autieri, M.M. Wysokiński, Phys. Rev. B 104, 024428 (2021).

[46] A.I. Liechtenstein, V.I. Anisimov, J. Zaanen, Phys. Rev. B 52, R5467(R) (1995).

[47] C. Autieri, E. Koch, E. Pavarini, Phys. Rev. B 89, 155109 (2014).

[48] M. Asa, G. Vinai, J.L. Hart et al., Phys. Rev. Mater. 2, 033401 (2018).

[49] M. Asa, C. Autieri, C. Barone, C. Mauro, S. Picozzi, S. Pagano, M. Cantoni, Phys. Rev. B 100, 174423 (2019).

[50] A. Jain, S.P. Ong, G. Hautier et al., APL Mater. 1, 11002 (2013).

[51] M. Asa, C. Autieri, R. Pazzocco, C. Rinaldi, W. Brzezicki, A. Stroppa, M. Cuoco, G. Varvaro, S. Picozzi, M. Cantoni, Phys. Rev. Res. 2, 043394 (2020).

[52] H. Xiang, C. Lee, H.-J. Koo, X. Gong, M.-H. Whangbo, Dalton Trans. 42, 823 (2013).

[53] B. Skubic, J. Hellsvik, L. Nordström, O. Eriksson, J. Phys. Condens. Matter 20, 315203 (2008).

[54] S. Toth, B. Lake, J. Phys. Condens. Matter 27, 166002 (2015). 